\title{
A High-Statistics Study of Low-Energy Neutrino-Nucleus Scattering In the NuMI Beam at Fermilab
}

\author{
Jorge G. Morfín \\ Fermi National Accelerator Laboratory, \\ P.O. Box 500, Batavia, IL,60510
}

\begin{abstract}
The NuMI Facility at Fermilab is providing an extremely intense beam of neutrinos for the MINOS Neutrino Oscillation Experiment. It an ideal place for a high statistics (anti)neutrino-nucleon/nucleus scattering experiments, and the MINER $v$ A experiment, a collaboration of elementary-particle and nuclear physicists, is planning to install a fully active fine-grained solid scintillator detector in this beam. The overall goals of the experiment are to measure absolute exclusive cross-sections, study nuclear effects in $v$ - A interactions and perform a systematic study of the resonance-DIS transition region including the extraction of high- $\mathrm{x}_{B j}$ parton distribution functions at low $\mathrm{Q}^{2}$.
\end{abstract}

Keywords: neutrino, nucleus, scattering

PACS: $13.15 .+\mathrm{g}$

\section{INTRODUCTION}

At Fermilab the NuMI neutrino facility, designed for the MINOS neutrino oscillation experiment [1], is based on the $120 \mathrm{GeV}$ protons from the Main Injector (MI) accelerator. The NuMI beam is yielding several orders of magnitude more events per $\mathrm{kg}$ of detector per unit of time than the earlier Tevatron neutrino beam did.

To take advantage of this intense beam, a collaboration of elementary particle and nuclear physics institutions named MINER $v$ A (Main Injector ExpeRiment: $v$ A) [2] was formed. The Fermilab PAC approved the MINER $v$ A proposal [3] in April 2004. The MINERvA project has been established at Fermilab and the collaboration is on track to start taking physics data in late 2008.

\section{THE FERMILAB NUMI FACILITY}

The Fermilab NuMI facility is made up of the technical beamline components including target, two magnetic focusing horns, evacuated decay pipe, monitoring devices, shielding and the underground facilities to contain the beamline components. A large, on-site experimental detector hall $\sim 100$ meters underground that contains the MINOS near detector and with sufficient extra space to house additional detectors is also part of NuMI.

The neutrino energy distribution of the NuMI beam can be chosen by changing the distance of the target and second horn with respect to the first horn, as in a zoom lens, or essentially continuously by simply varying the distance of target from the first horn and leaving the second horn in a fixed position. Depending on the chosen configuration, event rates will vary from $60 \mathrm{~K}$ to $520 \mathrm{~K}$ per ton of detector and $10^{20}$ protons on target (POT). The Main Injector is now delivering protons to MINOS at a rate equivalent to around $2.5 \times 10^{20} \mathrm{POT} / \mathrm{year}$ and will slowly build up to higher proton intensities. 
For the MINOS experiment the beamline will be operating mainly in its lowest possible neutrino energy configuration to be able to reach desired low values of $\delta m^{2}$. For the proposed NOvA experiment [4], the beam will be operating in the full ME configuration.

\section{OVERVIEW OF THE MINER $v$ A PHYSICS PROGRAM}

The MINERvA neutrino scattering experiment [3] in the NuMI beam offers a unique opportunity to study a broad spectrum of physics topics. Several have not previously been studied in any systematic way, while others have only few results that are compromised by large statistical and systematic errors. The high-statistics studies listed below are important for both the particle and nuclear physics communities, providing information complementary to JLab charged lepton studies in the same kinematic range:

- Precision measurement of the quasi-elastic neutrino-nucleus cross-section, including its $E_{v}$ and $q^{2}$ dependence, and study of the nucleon axial form factors.

- Determination of single- and double-pion production cross-sections in the resonance production region for both neutral-current and charged-current interactions, including a study of isospin amplitudes, measurement of pion angular distributions, isolation of dominant form factors, and measurement of the effective axial-vector mass.

- Clarification of the W (三 mass of the hadronic system) transition region where resonance production merges with neutrino deep-inelastic scattering, including tests of phenomenological characterizations of this transition such as quark/hadron duality.

- Precision measurement of coherent single-pion production cross-sections, with particular attention to target A dependence. Coherent $\pi^{0}$ production, via the neutral current, is a significant background for next-generation neutrino oscillation experiments seeking to observe $v_{\mu} \rightarrow v_{e}$ oscillation.

- Examination of nuclear effects in neutrino interactions, including final-state modifications in heavy nuclei, by employing carbon, iron and lead targets. MINER $v$ A will collect over 1.5M CC events each off carbon, iron and lead targets in addition to the scintillator sample. These effects play a significant role in neutrino oscillation experiments measuring $v_{\mu}$ disappearance as a function of $E_{v}$. It has recently been suggested [5] that, for a given $Q^{2}$, shadowing can occur at much lower energy transfer $(v)$ for neutrinos than for charged leptons. This effect is unaccounted for in neutrino event generators.

- Clarification of the role of nuclear effects as they influence the determination of $\sin ^{2} \theta_{W}$ via measurement of the ratio of neutral-current to charged-current cross-sections off different nuclei.

- Much-improved measurement of the parton distribution functions will be possible using a measurement of all six $v$ and $\bar{v}$ structure functions (with sufficient $\bar{v}$ running)

- Examination of the leading exponential contributions of perturbative QCD.

- Precision measurement of exclusive strange-particle production channels near threshold, thereby improving knowledge of backgrounds in nucleon-decay searches, determination of $V_{u s}$, and enabling searches for strangeness-changing neutral-currents and candidate pentaquark resonances. Measurement of hyperon-production cross-sections, including hyperon polarization, is feasible with exposure of MINER $v$ A to $\bar{v}$ beams.

These are worthy research topics in their own right, and improved knowledge in most is essential to minimizing systematic uncertainties in neutrino-oscillation experiments. 


\section{THE MINER $v$ A DETECTOR}

For MINER $v$ A to meet its physics goals, the detector must break new ground in the design of high-rate neutrino experiments. With final states as varied as high-multiplicity deep-inelastic reactions, coherent single- $\pi^{0}$ production and quasi-elastic neutrino scattering, the detector is a hybrid of a fully-active finegrained detector and a traditional calorimeter. A complete description of MINER $v \mathrm{~A}$ is found in the proposal [3] and CDR [6].

The MINER $v$ A detector is made up of a number of sub-detectors with distinct functions in reconstructing neutrino interactions. The fiducial volume for most analyses is the inner "Active Target" where all the material of the detector is the scintillator strips themselves. The scintillator detector does not fully contain events due to its low density and low $Z$, and therefore, the MINER $v$ A design surrounds the scintillator fiducial volume with sampling detectors. To construct these sampling detectors, the scintillator strips are intermixed with absorbers. For example, the side, upstream (US) and downstream (DS) electromagnetic calorimeters (ECALs) have lead foil absorbers. Surrounding the ECALs are the US and DS hadronic calorimeter (HCAL) where the absorbers are steel plates. The US ECAL and HCAL serve a dual purpose as high-A targets for the study of nuclear effects. On the sides of the detector the outer detector (OD) plays the role of the HCAL. Upstream of the detector is a veto of steel and scintillator strips to shield MINER $v$ A from incoming soft particles produced upstream in the hall.

The core active element will be extruded scintillator strips readout via wavelength-shifting fibers, similar in principle to the $\mathrm{K} 2 \mathrm{~K}$ SciBar[7]. Readout of the fibers will be done with multi-anode photomultiplier tubes (MAPMTs), connected to the wavelength shifting fibers via an optical cable system and housed in a light tight "optical box" mounted on the outer detector.

There are three distinct orientations of strips in the inner detector and veto, separated by $60^{\circ}$, and labeled X, U, V. A single module of MINER $v$ A has two X layers to seed two-dimensional track reconstruction, and one each of the $\mathrm{U}$ and $\mathrm{V}$ layers to reconstruct three-dimensional tracks.

Monte Carlo studies of this detector have confirmed that light-sharing with the triangular-shaped scintillator extrusions $(3.1 \mathrm{~cm}$ base and $1.7 \mathrm{~cm}$ height) yield reconstructed point resolution of just under $3 \mathrm{~mm}$. The electromagnetic $\left(\pi^{0}\right)$ energy resolution is $6 \% / \mathrm{sqrt}\left(E_{\text {em }}\right)$ while the hadron energy resolution is $4 \%+18 \% / \operatorname{sqrt}\left(E_{\text {had }}\right)$.

Acknowledgements

Operated by Fermi Research Alliance, LLC under Contract No. DE-AC02-07CD11359 with the United States Department of Energy.

\section{REFERENCES}

1. E. Ables et al. [MINOS Collaboration], FERMILAB-PROPOSAL-0875 and A. Marchionni [MINOS Collaboration], FERMILAB-CONF-05-429-AD-E

2. The MINER $v$ A Collaboration consists of groups from the following institutions: $U$ Athens, $U$ California/Irvine, U Dortmund, Fermilab, Hampton U, IL Inst. Tech., Inst. for Nuc. Research - Moscow, James Madison U, Jefferson Lab, U Nacional Ingeneria de Lima, N. Illinois U, Pontifica U Catolica de Lima, U Pittsburgh, U Rochester, Rutgers U, Tufts U, William and Mary U.

3. D. Drakoulakos et al. [Minerva Collaboration], arXiv:hep-ex/0405002. http://www.pas.rochester.edu/minerva/

4. D. S. Ayres et al. [NOvA Collaboration], arXiv:hep-ex/0503053.

5. S. Boyd, S. Kulagin, J. G. Morfin and R. Ransome, "Studying Neutrino-induced Nuclear Effects with the MINERvA Detector". MINERvA Note 700, September, 2004. http://www.pas.rochester.edu/minerva/+

6. D. Drakoulakos et al. [Minerva Collaboration], 23 December 2004, http://minerva.fnal.gov/

7. K. Nitta et al. (K2K SciBar), Nucl. Instrum. Meth. A536, 147 (2004) 